\title{
Monocarboxylate transporters (MCTs) and their role in hypothalamic glucosensing
}

\begin{abstract}
Monocarboxylate transporters (MCTs) are a family of transporters, which participate in the facilitated diffusion of lactate as well as the incorporation and release of several other metabolically important monocarboxylates, such as Pyruvate and ketone bodies. It has been established that lactate and ketone bodies have a role in energy balance. In the brain, lactate is an important oxidative energy substrate, and its intracerebroventricular (ICV) administration decreases food intake in rats. An opposite effect is produced by the ketone bodies. Several studies have suggested that the glucosensing mechanism is governed by a metabolic interaction between neurons and glial cells via lactate flux through MCTs. Hypothalamic glial cells (tanycytes) release lactate through MCT1 and MCT4. Moreover, orexigenic and anorexigenic neurons of the arcuate nucleus (AN) have high membrane immunoreactivity for MCT2, which is involved in lactate influx, suggesting that tanycytes indirectly control neuronal activity via glucose. This review will focus on MCT expression in the hypothalamus, a master regulation center for glucose homeostasis, and the evidence for a metabolic interaction between tanycytes and neuroendocrine neurons using monocarboxylates.
\end{abstract}

Keywords: monocarboxylate, tanycytes, neuroendocrine neurons, glucose, hypothalamus, glial cells
Volume 3 Issue 4 - 2016

\author{
Roberto Elizondo-Vega, Magdiel Salgado, \\ María de los Ángeles García-Robles \\ Department of Cell Biology, University of Concepción, Chile
}

Correspondence: María de los Ángeles García-Robles, Department of Cell Biology Laboratory of Cell Biology, Faulted of Biological Sciences, University of Concepción, Concepción, Chile, Tel 564I 2203805, Fax 564 I 2239687,

Emailmgarcia@udec.cl

Received: May 27, 2016 | Published: August 09, 2016
Abbreviations: MCT1, monocarboxylate transporter; AgRP, agouti-related peptide; NPY, neuropeptide y; POMC, proopiomelanocortin; ICV, intracerebroventricular; $3 \mathrm{~V}$, third ventricle; $\mathrm{AN}$, arcuate nucleus

\section{Introduction}

Monocarboxylate transporters (MCTs) have a high capacity to transport short-chain monocarboxylates, such as lactate, Pyruvate and the ketone bodies (KB), $\alpha$-hydroxybutyrate and acetoacetate, which have a role in energy balance. In the brain, lactate is an important oxidative energy substrate, ${ }^{1}$ and its intracerebroventricular (ICV) administration decreases both food intake and blood glucose levels in rats. ${ }^{2}$ Ketone bodies also affect food intake ${ }^{3,4}$ and body weight in an opposing manner. ${ }^{5}$ In the hypothalamus, a brain region involved in energy balance, it has been shown that MCTs have a particular cellular distribution, which supports the transfer of monocarboxylates between glia and neurons. MCT1 and MCT4 are expressed in the main glial cells (tanycytes) in the basal hypothalamus. $\alpha$ and $\beta$-tanycytes are specialized ependymal cells that bridge the cerebrospinal fluid (CSF) and neuroendocrine neurons localized in the arcuate nucleus $(\mathrm{AN})^{6}$ (Figure 1). Specifically, tanycyte processes that are MCT1- and MCT4-positive are in direct contact with MCT2 positives neurons that synthesize and release neuropeptides that control food intake, such as neuropeptide Y (NPY)-, agouti-related protein (AgRP), and proopiomelanocortin (POMC). ${ }^{7,8}$ MCTs comprise a family of fourteen members (MCT1-14) distributed in a wide variety of tissues; ${ }^{9-11}$ they use the electrochemical gradient of protons to translocate monocarboxylates and protons at a 1:1 ratio in the same direction. ${ }^{12,13}$ MCT1 has the widest selectivity, and may transport several short chain fatty acids, such as acetate, butyrate, lactate or Pyruvate. ${ }^{14}$ Brain localization studies using immunocytochemistry, in situ hybridization and Immunoblotting have determined that MCT1 is predominantly a glial transporter, expressed in cortical astrocytes, ${ }^{15-22}$ hippocampal astrocytes, ${ }^{23,24}$ astrocytes of the supraoptic nucleus, ${ }^{21}$ choroid plexus, ependymal ciliated cells, ${ }^{22,25}$ endothelial cells and pericytes. ${ }^{17,20}$ In vitro neuronal expression of MCT1 has also been reported and recently in NPY-expressing neurons in situ. ${ }^{3}$ MCT2 has a much higher affinity for Pyruvate although it also transports lactate and ketone bodies. ${ }^{14}$. MCT2 localization is mainly at the neuronal level in the cortical neurons, ${ }^{26}$ and cerebellar Purkinje cells, ${ }^{27}$ in the postsynaptic density of the asymmetrical excitatory synapses of the hippocampus ${ }^{28}$ Purkinje neuron cell bodies. ${ }^{27}$ In addition, MCT2 was detected in marginal glia, ependymal cells ${ }^{18}$ and the vascular processes of astrocytes. ${ }^{18,22}$ Recently, we have identified MCT2 localization in anorexigenic and orexigenic neurons of the AN. ${ }^{7}$ In accordance with a participation in energy balance, it has been reported that insulin stimulates the expression of MCT2 in cultured neurons..$^{29}$ MCT3 expression has only been characterized using Northern blot and immunohistochemistry studies and found in the basolateral membrane of the choroid plexus. ${ }^{30}$ MCT4, which transports lactate and ketone bodies and has a very low affinity for Pyruvate, was detected exclusively in glial cells, particularly in the Bergmann glia and astrocytes of the molecular and granular layers of the cerebellum..$^{21,24,27,31}$ In addition, MCT4 expression was detected in astrocytes of the hippocampus, corpus callosum and cerebral corte $\mathrm{x}^{24}$ as well as in ciliated ependymal cells of the paraventricular nucleus. ${ }^{22}$ We have described the localization of MCT4 in tanycyte processes that contact distal neurons in the AN. ${ }^{6}$ Therefore, the presence of MCT1 and MCT4 in tanycytic basal processes and MCT2 expression in AN neurons suggest that lactate could be transferred between both cellular types as a signal derived from glucose for regulating energy balance and food intake. In support of this model, it has been demonstrated that glucose increases intracellular $\mathrm{Ca}^{2+}$ concentration $\left(\left[\mathrm{Ca}^{2+}\right]_{\mathrm{i}}\right)$ in cultured tanycytes, a response that is oxidative phosphorylation-independent but can be diminished through the inhibition of glucokinase, a critical glucosensor enzyme expressed in tanycytes. ${ }^{32-34}$ Interestingly, 
this mechanism involves the opening of hemi channels $(\mathrm{HC})$, and pharmacological inhibitors of $\mathrm{HC}$ abolish the response to glucose, but do not affect the efflux of lactate. ${ }^{6,33,35}$ Several studies have shown that ICV administration of lactate decreases food intake and stimulates pancreatic insulin secretion. ${ }^{36,37}$ Moreover, recent studies demonstrate that glial connexins are required for hypothalamic glucosensing, suggesting that glial glucose metabolism is needed for centrally mediated glucose-induced insulin secretion as well as suppression of food intake. ${ }^{36}$ Brain use of ketone bodies in normoglycemia is very low, however in fasting or high-fat diets this increases considerably. ${ }^{4}$ Ketone bodies, mainly produced in liver, are incorporated into the brain by MCT1, which is expressed in endothelial cells of cerebral blood vessels. ${ }^{38-40}$ Interestingly, the hypothalamus, which is a key player in brain sensing of metabolic signals, presents higher ketone body metabolism than other brain areas (Figure 2) ${ }^{41}$ Furthermore, it has been shown that astrocytes in culture could release ketone bodies, generated by the catabolism of fatty acids..$^{42}$ It is also possible that ketone bodies can be generated by glial cells of the hypothalamus (astrocytes or tanycytes). In support of this notion, the presence of numerous lipid droplets and the expression of enzymes involved in lipid metabolism have been demonstrated in tanycytes using histological and immunohistochemical analyses. ${ }^{43}$ Therefore ketone bodies release could be realised by glial cells and/or peripheral by hepatocytes. Alternatively, the neuronal effects of ketone bodies could be mediated by MCTs or through hydroxy-carboxylic acid receptors, which have been located in several brain regions ${ }^{44}$ and which are Gicoupled receptors. ${ }^{45-47}$ The mechanisms that allow to ketone bodies to modulate the activity of neurons that control food intake still remain to be elucidated.

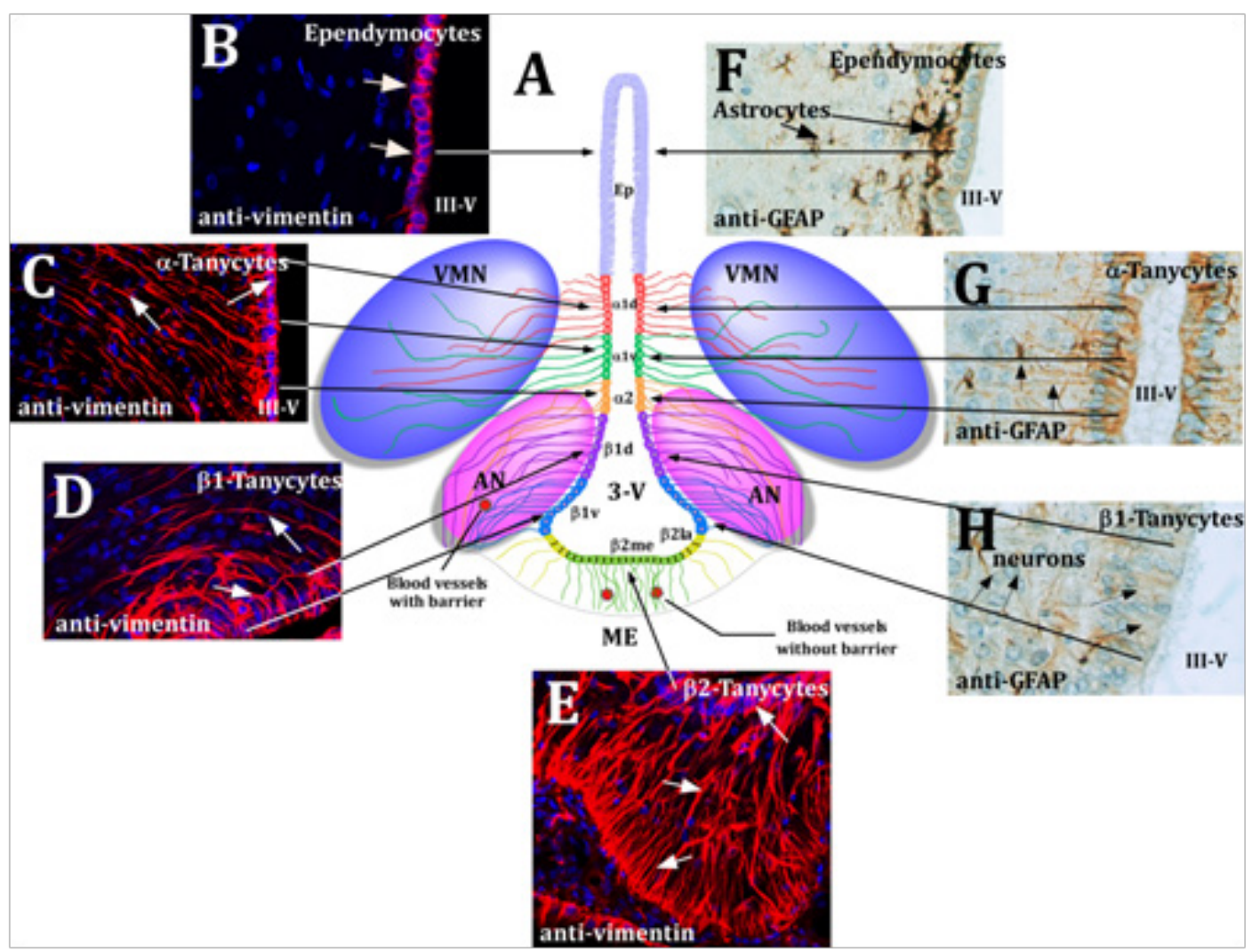

Figure I Schematic representation of the hypothalamus

(A) Frontal view of the hypothalamus.

I. Ciliated Ependymocytes;

2. $\alpha I$ and $\alpha 2$-tanycytes contacting neurons of the $\mathrm{AN}, \mathrm{VMN}$ and blood vessels;

3. $\beta$ I-tanycytes line the infundibular recess, and their basal projections reach the lateral regions of the ME and the AN;

4. $\beta 2$-tanycytes cover the floor of the III-V and extend their projections inside the ME.

(B-E) Immunohistochemical analysis of the hypothalamic tanycytes. Representative confocal images of frontal hypothalamic sections immunostained with antivimentin antibody. Ependymocyte cells (B), a (C), $\beta$ I (D) $\beta 2$-tanycytes (E) are positive for the expression of vimentin.

(F-H) Immunohistochemical analysis of hypothalamic glial cells. The hypothalamic sections were incubated with anti-GFAP and the immunoreaction was developed using the PAP method.Astrocytes, localized under the ependimary cells (F) and $\alpha$-tanycytes (G) are positive for GFAP.A weaker reaction was found in $\beta$ I-Tanycytes $(\mathbf{H})$.

AN, arcuate nucleus; VMN, ventromedial nucleus; Ep, ependymocytes; 3-V, third ventricle.

Modificated from Elizondo-Vega et al. ${ }^{8}$ 


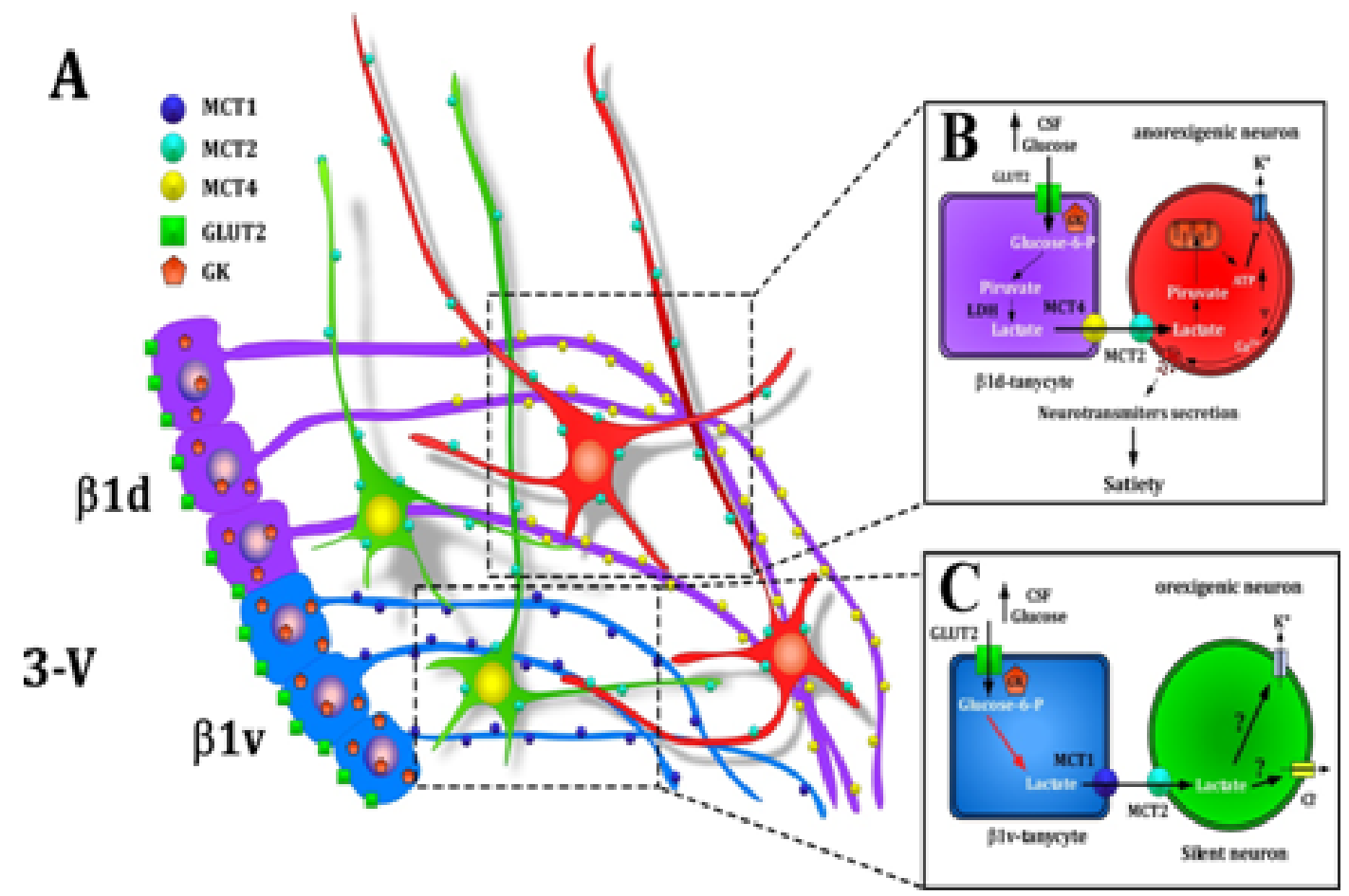

Figure 2 Model of cerebral glucose sensing based on the metabolic interaction between $\beta$ Id-tanycytes or $\beta$ Iv-tanycytes and neurons.

A. Schematic representation of the location of MCT4 (yellow) in $\beta$ Id-tanycytes processes (purple), MCTI (blue) in $\beta$ Iv-tanycytes processes (light blue), and MCT2 (light green) in orexigenic (green) and anorexigenic (red) neurons of the AN.

B. Schematic overview of the model of glial-neuronal interaction based on the transfer of lactate proposed for cerebral glucose sensing between GE neurons and tanycytes.

C. Scheme based on proposed interaction between $\beta$ Iv-tanycytes and orexigenic neurons compared to the increase in glucose concentration in the CSF $3-\mathrm{V}$, third ventricle; BId and BIv, tanycytes; CSF, cerebral spinal fluid; GK, glucokinase; LDH, lactate dehydrogenase. Modificated from Elizondo-Vega et al. ${ }^{8}$

\section{Conclusion}

MCTs mediate the release of lactate from tanycytes and its incorporation into neurons, which could generate satiety when glucose concentrations rise. In contrast, fasting could favor the incorporation of ketone bodies through the blood-brain barrier or their release from glial cells, activating neurons that induce hunger or inhibiting satiety neurons. Current literature supports the participation of MCTs in food intake, which is relevant under low- and high-glucose conditions.

\section{Acknowledgements}

None.

\section{Conflict of interest}

The author declares no conflict of interest.

\section{References}

1. Magistretti PJ, Pellerin L. Cellular mechanisms of brain energy metabolism and their relevance to functional brain imaging. Philos Trans R Soc Lond B Biol Sci. 1999;354(1387):1155-1163.

2. Lam CK, Chari M, Wang PY, et al. Central lactate metabolism regulates food intake. Am J Physiol Endocrinol Metab. 2008;295(2):E491-E496.

3. Carneiro L, Geller S, Fioramonti X, et al. Evidence for hypothalamic ketone body sensing: impact on food intake and peripheral metabolic responses in mice. Am J Physiol Endocrinol Metab. 2016;310(2):E103E115.
4. Hawkins RA, Mans AM, Davis DW. Regional ketone body utilization by rat brain in starvation and diabetes. Am J Physiol. 1986;250(2 Pt 10):E169-E178.

5. Iwata $\mathrm{K}$, Kinoshita $\mathrm{M}$, Yamada $\mathrm{S}$, et al. Involvement of brain ketone bodies and the noradrenergic pathway in diabetic hyperphagia in rats. $J$ Physiol Sci. 2011;61(2):103-113.

6. Cortés-Campos C, Elizondo R, Llanos P, et al. MCT expression and lactate influx/efflux in tanycytes involved in glia-neuron metabolic interaction. PLoS One. 2011;6(1):e16411.

7. Cortés-Campos C, Elizondo R, Carril C, et al. MCT2 expression and lactate influx in anorexigenic and orexigenic neurons of the arcuate nucleus. PLoS One. 2013;8(4):e62532.

8. Elizondo-Vega R, Cortes-Campos C, Barahona MJ, et al. The role of tanycytes in hypothalamic glucosensing. $J$ Cell Mol Med. 2015;19(7):1471-1482.

9. Halestrap AP. The monocarboxylate transporter family - Structure and functional characterization. IUBMB Life. 2012;64(1):1-9.

10. Halestrap AP. The SLC16 gene family - structure, role and regulation in health and disease. Mol Aspects Med. 2013;34(2-3):337-349.

11. Halestrap AP. Monocarboxylic acid transport. Compr Physiol. 2013;3(4):1611-1643.

12. Gladden LB. Lactate metabolism:a new paradigm for the third millennium. J Physiol. 2004;558(Pt 1):5-30.

13. Halestrap AP, Price NT. The proton-linked monocarboxylate transporter (MCT) family: structure, function and regulation. Biochem J. 1999;343(Pt 2):281-299. 
14. Carneiro L, Pellerin L. Monocarboxylate transporters: new players in body weight regulation. Obes Rev. 2015;16(Suppl 1):55-66.

15. Broer S, Rahman B, Pellegri G, et al. Comparison of lactate transport in astroglial cells and monocarboxylate transporter 1 (MCT 1) expressing Xenopus laevis oocytes. Expression of two different monocarboxylate transporters in astroglial cells and neurons. $J$ Biol Chem. 1997;272(48):30096-30102.

16. Debernardi R, Pierre K, Lengacher S, Cell-specific expression pattern of monocarboxylate transporters in astrocytes and neurons observed in different mouse brain cortical cell cultures. $J$ Neurosci Res 2003;73(2):141-155

17. Gerhart DZ, Enerson BE, Zhdankina OY, et al. Expression of monocarboxylate transporter MCT1 by brain endothelium and glia in adult and suckling rats. Am J Physiol. 1997;273(1 Pt 1):E207-E113.

18. Gerhart DZ, Enerson BE, Zhdankina OY, et al. Expression of the monocarboxylate transporter MCT2 by rat brain glia. Glia. 1998;22(3):272-281.

19. Hanu R, McKenna M, O’Neill A, et al. Monocarboxylic acid transporters, MCT1 and MCT2, in cortical astrocytes in vitro and in vivo. Am J Physiol Cell Physiol. 2000;278(5):C921-C930.

20. Leino RL, Gerhart DZ, Drewes LR. Monocarboxylate transporter (MCT1) abundance in brains of suckling and adult rats: a quantitative electron microscopic immunogold study. Brain Res Dev Brain Res. $1999 ; 113(1-2): 47-54$

21. Pellerin L, Bergersen LH, Halestrap AP, et al. Cellular and subcellular distribution of monocarboxylate transporters in cultured brain cells and in the adult brain. $J$ Neurosci Res. 2005;79(1-2):55-64.

22. Pierre K, Pellerin L, Debernardi R, et al. Cell-specific localization of monocarboxylate transporters, MCT1 and MCT2, in the adult mouse brain revealed by double immunohistochemical labeling and confocal microscopy. Neuroscience. 2000;100(3):617-627.

23. Assaf HM, Ricci AJ, Whittingham TS, et al. Lactate compartmentation in hippocampal slices: evidence for a transporter. Metab Brain Dis. 1990;5(3):143-154.

24. Rafiki A, Boulland JL, Halestrap AP, et al. Highly differential expression of the monocarboxylate transporters MCT2 and MCT4 in the developing rat brain. Neuroscience. 2003;122(30):677-688.

25. Koehler-Stec EM, Simpson IA, Vannucci SJ, et al. Monocarboxylate transporter expression in mouse brain. Am J Physiol. 1998;275(3 P 1):E516-E524.

26. Pierre K, Pellerin L. Monocarboxylate transporters in the centra nervous system: distribution, regulation and function. $J$ Neurochem. 2005;94(1):1-14.

27. Bergersen L, Waerhaug O, Helm J, et al. A novel postsynaptic density protein: the monocarboxylate transporter MCT2 is co-localized with delta-glutamate receptors in postsynaptic densities of parallel fiberPurkinje cell synapses. Exp Brain Res. 2001;136(4):523-534.

28. Bergersen LH, Magistretti PJ, Pellerin L. Selective postsynaptic co-localization of MCT2 with AMPA receptor GluR2/3 subunits at excitatory synapses exhibiting AMPA receptor trafficking. Cereb Cortex. 2005;15(4):361-370.

29. Chenal J, Pierre K, Pellerin L. Insulin and IGF-1 enhance the expression of the neuronal monocarboxylate transporter MCT2 by translational activation via stimulation of the phosphoinositide 3kinase-Akt-mammalian target of rapamycin pathway. Eur J Neurosci. 2008;27(1):53-65
30. Philp NJ, Yoon H, Lombardi L. Mouse MCT3 gene is expressed preferentially in retinal pigment and choroid plexus epithelia. Am J Physiol Cell Physiol. 2001;280(5):C1319-C1326.

31. Bergersen L, Rafiki A, Ottersen OP. Immunogold cytochemistry identifies specialized membrane domains for monocarboxylate transport in the central nervous system. Neurochem Res. 2002;27(1-2):89-96.

32. Millan C, Martinez F, Cortes-Campos C, et al. Glial glucokinase expression in adult and post-natal development of the hypothalamic region. ASN Neuro. 2010;2(3):e00035.

33. Orellana JA, Saez PJ, Cortes-Campos C, et al. Glucose increases intracellular free $\mathrm{Ca}(2+)$ in tanycytes via ATP released through connexin 43 hemichannels. Glia. 2012;60(1):53-68.

34. Salgado M, Tarifeno-Saldivia E, Ordenes P, et al. Dynamic localization of glucokinase and its regulatory protein in hypothalamic tanycytes. PLoS One. 2014;9(4):e94035.

35. Frayling C, Britton R, Dale N. ATP-mediated glucosensing by hypothalamic tanycytes. J Physiol. 2011;589(Pt 9):2275-2286.

36. Allard C, Carneiro L, Collins SC, et al. Alteration of hypothalamic glucose and lactate sensing in $48 \mathrm{~h}$ hyperglycemic rats. Neurosci Lett. $2013 ; 534: 75-79$.

37. Cha SH, Lane MD. Central lactate metabolism suppresses food intake via the hypothalamic AMP kinase/malonyl-CoA signaling pathway. Biochem Biophys Res Commun. 2009;386(1):212-216.

38. Leino RL, Gerhart DZ, Duelli R, et al. Diet-induced ketosis increases monocarboxylate transporter (MCT1) levels in rat brain. Neurochem Int. 2001;38(6):519-527.

39. Paoli A, Cenci L, Grimaldi KA. Effect of ketogenic Mediterranean diet with phytoextracts and low carbohydrates/high-protein meals on weight, cardiovascular risk factors, body composition and diet compliance in Italian council employees. Nutr J. 2011;10:112.

40. Veech RL. The therapeutic implications of ketone bodies: the effects of ketone bodies in pathological conditions: ketosis, ketogenic diet, redox states, insulin resistance, and mitochondrial metabolism. Prostaglandins Leukot Essent Fatty Acids. 2004;70(3):309-319.

41. Hawkins RA, Biebuyck JF. Ketone bodies are selectively used by individual brain regions. Science. 1979;205(4403):325-327.

42. Blazquez $\mathrm{C}$, Woods $\mathrm{A}$, de Ceballos $\mathrm{ML}$, et al. The AMP-activated protein kinase is involved in the regulation of ketone body production by astrocytes. J Neurochem. 1999;73(40):1674-1682.

43. Akmayev IG, Popov AP. Morphological aspects of the hypothalamichypophyseal system. VII. The tanycytes: Their relation to the hypophysea adrenocorticotrophic function. An ultrastructural study. Cell Tissue Res. 1977;180(2):263-282.

44. Lauritzen KH, Morland C, Puchades M, et al. Lactate Receptor Sites Link Neurotransmission, Neurovascular Coupling, and Brain Energy Metabolism. Cerebral Cortex. 2014;24(10):2784-2795

45. Liu C, Wu J, Zhu J, et al. Lactate inhibits lipolysis in fat cells through activation of an orphan G-protein-coupled receptor, GPR81. J Biol Chem. 2009;284(5):2811-2822.

46. Tunaru S, Kero J, Schaub A, et al. PUMA-G and HM74 are receptors for nicotinic acid and mediate its anti-lipolytic effect. Nat Med. 2003;9(3):352-355.

47. Wise A, Foord SM, Fraser NJ, et al. Molecular identification of high and low affinity receptors for nicotinic acid. J Biol Chem. 2003;278(11):98699874. 